EPJ Web of Conferences 53, 07005 (2013)

DOI: $10.1051 /$ epjconf/20135307005

(C) Owned by the authors, published by EDP Sciences, 2013

\title{
Measurement of the proton-air cross-section with the Pierre Auger Observatory
}

\author{
Ralf Ulrich ${ }^{1, \mathrm{a}}$ for the Pierre Auger Collaboration ${ }^{2, \mathrm{~b}}$ \\ ${ }^{1}$ Karlsruhe Institute of Technology, Germany \\ ${ }^{2}$ Observatorio Pierre Auger, Malargüe, Argentina
}

\begin{abstract}
We present the procedure to measure the proton-air cross-section at a center-of-mass energy per nucleon of $57 \mathrm{TeV}$ developed by the Pierre Auger Collaboration. The conversion from proton-air to inelastic proton-proton cross-section with an extended Glauber calculation is discussed. The systematic uncertainties of the analysis are summarized and the final result compared to accelerator data and model predictions.
\end{abstract}

\section{INTRODUCTION}

The growth of hadronic cross-sections towards ultra-high energies reflects changes in the internal structure of the colliding particles. Due to the nature of QCD it is still not possible to calculate hadronic cross-sections at ultra-high energies from first principles. It is thus of paramount importance to measure hadronic cross-sections in order to guide the development of phenomenological models needed to describe secondary multiparticle production at ultra-high energies. Such models are crucial, for example, for a detailed interpretation of available cosmic-ray air-shower data in terms of the primary mass composition. Without a better understanding of the cosmic-ray mass composition it will be very difficult to distinguish between different scenarios of cosmic-ray acceleration and propagation.

We present the measurement of the proton-air cross-section with hybrid data of the Pierre Auger Observatory [1]. The Pierre Auger Observatory is located close to the town of Malargüe in Argentina. It consist of about 1660 water Cherenkov particle detector spread over a surface area of $3000 \mathrm{~km}^{2}$. During clear and moonless nights the atmosphere on top of the surface array is overlooked by 27 fluorescence telescopes. This analysis is based on the observation of the longitudinal air-shower development with the telescopes. By combining the timing data of the surface detectors with that of the fluorescence telescopes a very precise reconstruction of the geometry is achieved [2]. This technique is called "hybrid" reconstruction. It allows us a very accurate measurement of $X_{\max }$, the position at which an air-shower deposits the maximum energy per unit of mass of atmosphere traversed [3].

With the precise observation of longitudinal air-shower fluctuations it is possible to achieve high sensitivity to the mean free path of the primary cosmic-ray particles and thus to the cross-section. This technique is well known and has been first applied by the Fly's Eye experiment [4].

We also discuss systematic uncertainties and limitations of the cross-section measurement. In a last step, the proton-air cross-section measurement is converted into a proton-proton cross-section. This conversion makes use of a Glauber calculation, which has been extended to account for inelastic screening effects.

\footnotetext{
${ }^{a}$ e-mail: ralf .ulrich@kit.edu

bFor the full authorlist see Appendix "Collaborations" in this volume.

This is an Open Access article distributed under the terms of the Creative Commons Attribution License 2.0, which permits unrestricted use, distribution, and reproduction in any medium, provided the original work is properly cited.
} 


\section{EPJ Web of Conferences}

\section{ANALYSIS METHOD}

Event-by-event fluctuations of $X_{\max }$ can be measured with the Pierre Auger Observatory. These fluctuations are directly related to the fluctuation of the depth of the first interaction in the atmosphere. In order to determine the hadronic cross-section for the first interaction, the additional fluctuations induced during the shower development, following the first interaction, have to be subtracted. This is a model dependent procedure [5]. Thus, we are performing the analysis in several steps. We first perform a measurement of air-shower fluctuations. Since it is known that the primary mass composition has a major impact on air-shower fluctuations, we reduce the sensitivity to the mass composition as much as possible. Firstly, we use only data in the energy range $10^{18}-10^{18.5} \mathrm{eV}$, where our data is best described with a significant fraction of protons [3]. Secondly, we use only the exponential slope of the tail of the $X_{\max }$ distribution at large depths, $\Lambda_{\eta}$, since heavier primary particles are less penetrating with respect to protons. The remaining dependence on the mass composition and also of a possible photon contamination [6] is evaluated with simulations and included in the systematic uncertainties. The range in slant depth of the analysis is defined to contain the $20 \%$ most deeply penetrating events, which is a definition that can be easily applied also on simulation level.

\section{MEASUREMENT OF $\Lambda_{\eta}$}

The measurement of $\Lambda_{\eta}$ is a challenging task: the tail of the distribution of $X_{\max }$ is characterised by the combination of low statistics and experimental acceptance inefficiencies. We apply quality and fiducial volume cuts to maximise our event statistics while, at the same time, minimizing the impact of acceptance effects.

The impact of the telescope acceptance on the $X_{\max }$ distribution is well understood and can be studied by using data (see [15]) and with detailed Monte Carlo simulations of the shower detection process. We use the strategy developed for the measurement of the $\left\langle X_{\max }\right\rangle$ and $\operatorname{RMS}\left(X_{\max }\right)[3,15]$ to extract a data sample that has an unbiased $X_{\max }$ distribution. First we evaluate the overall shape and location of the measured $X_{\max }$ distribution with very strict fiducial volume cuts. This is used to define the slant depth range containing the $20 \%$ most deeply penetrating events. In a last selection step the event statistics is maximised by reducing the range of the fiducial volume selection to match exactly the slant depth range relevant for further analysis.

In Fig. 1 (left) we show the selected data set of 3082 events and the result of an unbinned maximum likelihood fit of an exponential function over the range 768 to $1004 \mathrm{~g} / \mathrm{cm}^{2}$. Values of $\Lambda_{\eta}$ have been calculated for modified event selections and for different ranges of atmospheric depths. It is found that the changes in $\Lambda_{\eta}$ lie within the statistical uncertainties. The re-analyses of the data for changes of fiducial event selection, modified values of $\eta$ and for different ranges of atmospheric depths yield changes of $\Lambda_{\eta}$ that are distributed around zero with a root-mean-square of $1.6 \mathrm{~g} / \mathrm{cm}^{2}$. We use this rootmean-square as an estimate of the systematic uncertainties associated to the measurement. This yields

$$
\Lambda_{\eta}=\left[55.8 \pm 2.3_{\text {stat }} \pm 1.6_{\text {syst }}\right] \mathrm{g} / \mathrm{cm}^{2}
$$

with the average energy of these events being $10^{18.24} \mathrm{eV}$. This corresponds to a center-of-mass energy of $\sqrt{s}=[57 \pm 0.3$ (stat) $] \mathrm{TeV}$ in proton-proton collisions.

\section{DETERMINATION OF $\sigma_{\mathrm{p}-\mathrm{air}}$}

The determination of the proton-air cross-section for particle production requires the use of air-shower simulations, which inherently introduces some dependence on model assumptions. We emulate the measurement of $\Lambda_{\eta}$ with Monte Carlo simulations to derive predictions of the slope, $\Lambda_{\eta}^{\mathrm{MC}}$. It is known from previous work that the values of $\Lambda_{\eta}^{\mathrm{MC}}$ are directly linked to the hadronic cross-sections used in the simulations [4]. Accordingly we can explore the effect of changing cross-sections empirically by 

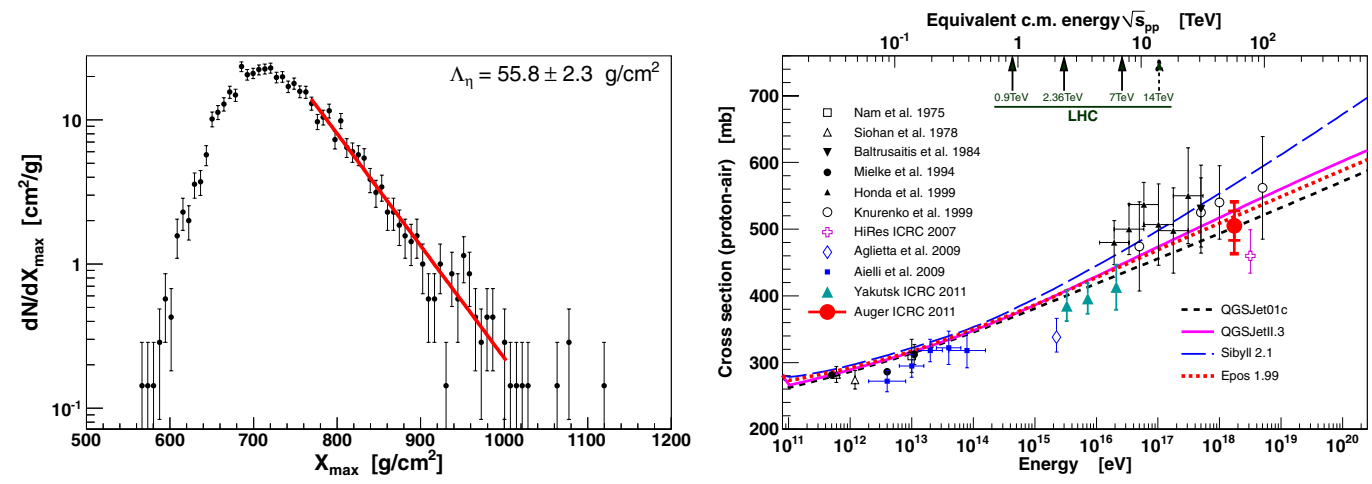

Figure 1. Left panel: unbinned likelihood fit to obtain $\Lambda_{\eta}$ (thick line). The $X_{\max }$-distribution is unbiased by the fiducial geometry selection applied in the range of the fit. Right panel: resulting $\sigma_{p-\text { air }}^{\text {prod }}$ compared to other measurements (see [7-14] for references) and model predictions. The inner error bars are statistical, while the outer are the sum of statistic and systematic uncertainties in quadrature.

multiplying all hadronic cross-sections input to the simulations by an energy-dependent factor [16]

$$
f\left(E, f_{19}\right)=1+\left(f_{19}-1\right) \frac{\lg \left(E / 10^{15} \mathrm{eV}\right)}{\lg \left(10^{19} \mathrm{eV} / 10^{15} \mathrm{eV}\right)},
$$

where $E$ denotes the shower energy and $f_{19}$ is the factor by which the cross-section is rescaled at $10^{19} \mathrm{eV}$. This factor is unity below $10^{15} \mathrm{eV}$ reflecting the fact that measurements of the crosssection at the Tevatron were used to tune the interaction models. This technique of modifying the original predictions of the cross-sections during the simulation process assures a smooth transition from accelerator data up to the energies of our analysis.

The proton-air cross-sections for particle production derived from data using QGSJet01, QGSJetII, SIBYLL and EPOS are 523.7, 502.9, 496.7 and $497.7 \mathrm{mb}$ respectively, with the statistical uncertainty for each of these values being $22 \mathrm{mb}$. The difference of these cross-sections from the original model predictions are $<5 \%$, with the exception of the result obtained with the SIBYLL model, which is $12 \%$ smaller than the original SIBYLL prediction. We use the maximum deviations derived from using the four models, relative to the average result of $505 \mathrm{mb}$, to estimate a systematic uncertainty of $(-8,+19) \mathrm{mb}$ related to the difficulties of modelling high energy interactions. This procedure relies on the coverage of the underlying theoretical uncertainties by the available models. For example diffraction, fragmentation, inelastic intermediate states, nuclear effects, QCD saturation, etc. are all described at different levels using different phenomenological, but self-consistent, approaches in these models. The true range of the uncertainties cannot be estimated with these models. Furthermore, certain features of hadronic particle production, such as the multiplicity, elasticity and pion-charge ratio, have an especially important impact on air-shower development [17, 18]; of these we found that only the elasticity can have a relevant impact on $\Lambda_{\eta}$. The previously identified systematic uncertainty of $(-8,+19)$ mb induced by the modelling of hadronic interactions, corresponds to the impact of modifying the elasticity within $\pm(10-25) \%$ in the models.

The systematic uncertainty of $22 \%$ [19] in the absolute value of the energy scale leads to systematic uncertainties of $7 \mathrm{mb}$ in the cross-section and $6 \mathrm{TeV}$ in the center-of-mass energy. Furthermore, the procedure to obtain $\sigma_{p \text {-air }}^{\text {prod }}$ from the measured $\Lambda_{\eta}$ depends on additional parameters. By varying the energy distribution, energy and $X_{\max }$ resolution in the simulations, we find that related systematic changes of the value of $\sigma_{p \text {-air }}^{\text {prod }}$ are distributed with a root-mean-square of $7 \mathrm{mb}$ around zero. We use the root-mean-square as estimate of the systematic uncertainties related to the conversion of $\Lambda_{\eta}$ to $\sigma_{p \text {-air }}^{\text {prod }}$. 
The presence of photons in the primary beam would bias the measurement. The average $X_{\max }$ of showers produced by photons at the energies of interest is more than $100 \mathrm{~g} / \mathrm{cm}^{2}$ deeper in the atmosphere than that of protons. However, observational limits on the fraction of photons are $<0.5 \%[6,20]$. With simulations we find that the possible under-estimation of the cross-section - if photons were present in the data sample at this level - is less than $10 \mathrm{mb}$.

There is a partial ambiguity between the amount of helium in the data set and the interpretation of the $X_{\max }$ distributions in terms of proton-air cross-section. From simulations we find that $\sigma_{p \text {-air }}^{\text {prod }}$ is overestimated by 12, 30 and $80 \mathrm{mb}$ for percentages of helium in the data of 10, 25 and 50\% respectively. Lack of knowledge of the helium fraction is the dominant source of systematic uncertainty. As the helium fraction is not known, we include a systematic uncertainty related to a helium fraction of $25 \%$ in the following. In the extreme case, were the cosmic-ray composition to be almost entirely helium, the analysis would over-estimate the proton-air cross-section by 300 to $500 \mathrm{mb}$. Given the constraints from accelerator data at lower energies and typical model assumptions, this extreme scenario is not realistic.

We also find that the nuclei of the CNO-group introduce only a negligible bias for fractions up to $\sim 50 \%$, and accordingly we assign no uncertainty in the cross-section due to these or heavier nuclei.

We summarise our results as

$$
\sigma_{p-\text { air }}^{\text {prod }}=\left[505 \pm 22(\text { stat })_{-36}^{+28}(\text { sys })\right] \mathrm{mb}
$$

at a center-of-mass energy of $[57 \pm 0.3$ (stat) \pm 6 (sys) $] \mathrm{TeV}$. For a more detailed discussion of the included systematic uncertainties see Ref. [5]. In Fig. 1 (right) we compare this result with model predictions and other measurements.

It is one of the prime aims of our analysis to have the smallest possible sensitivity to a non-proton component, and to perform a detailed systematic analysis on the uncertainties related to the mass composition. We also use, for the first time, all hadronic interaction models currently available for the estimation of model-related systematic effects. Furthermore, by using Eq. (1), we derive a crosssection corresponding to a smooth interpolation from the Tevatron measurement to our analysis, with no inconsistencies as in earlier approaches.

\section{COMPARISON TO ACCELERATOR DATA}

For the purpose of making comparisons with accelerator data we calculate the inelastic and total proton-proton cross-sections using the Glauber model [32] extended by a two-channel implementation of inelastic intermediate states [22] to account for diffraction dissociation [33]. The first channel corresponds to $\mathrm{p} \rightarrow \mathrm{p}$ scattering and has an amplitude of $\Gamma_{\mathrm{pp}}$, while the amplitude for the other channel is $\Gamma_{\mathrm{pp}^{*}}=\lambda \Gamma_{\mathrm{pp}}$ and corresponds to the (de)excitation of a short lived intermediate state, $\mathrm{p} \leftrightarrow \mathrm{p}^{*}$. The parameter $\lambda$ is related to the ratio of the single-diffractive cross-section, $\sigma_{\mathrm{SD}}$, and the elastic crosssection, $\sigma_{\mathrm{el}}$, as $\lambda^{2}=\sigma_{\mathrm{SD}} /\left(2 \sigma_{\mathrm{el}}\right)$. However, there is a normalization freedom in choosing $\lambda$ (c.f. Fig. 2 , left) as it is not defined by the theory up to which diffractive mass, $\xi_{\max }=M_{\mathrm{D}}^{2} / s$, the state $\mathrm{p}^{*}$ contributes to the screening and, thus, up to which $\xi_{\max }$ one has to integrate $\sigma_{\mathrm{SD}}$. Using different parameterizations of available accelerator data for both $\sigma_{\mathrm{SD}}[34]$ and $\sigma_{\mathrm{el}}$ we tune this range in $\xi_{\max }$ to get a good mapping from proton-proton to measured proton-carbon cross-sections, see Fig. 2 (right). Note also, that the standard Glauber model cannot predict proton-carbon from proton-proton well. Within the extended Glauber model we identify an optimal value of $\lambda=0.5 \pm 0.15$ at $\sqrt{s}=57 \mathrm{TeV}$. Details will be given elsewhere [27].

This Glauber calculation is model-dependent since neither the parameters nor the physical processes involved are known accurately at cosmic-ray energies. In particular, this applies to the elastic slope parameter, $B_{\mathrm{el}}$, (defined by $\mathrm{d} \sigma_{\mathrm{el}} / \mathrm{d} t \propto \exp \left(-|t| B_{\mathrm{el}}\right)$ for very small $t$ ), the correlation of $B_{\mathrm{el}}$ to the crosssection, and the cross-section for diffractive dissociation. For the example of $\sigma_{p p}^{\text {inel }}$, the correlation of $B_{\mathrm{el}}$ with the cross-section is shown in Fig. 3 (left) for $\lambda=0.5$. We have used the same four hadronic interaction models to determine the uncertainty band of the $B_{\mathrm{el}}-\sigma_{p p}^{\text {inel }}$ correlation. Recent cross-section 


\section{UHECR 2012}
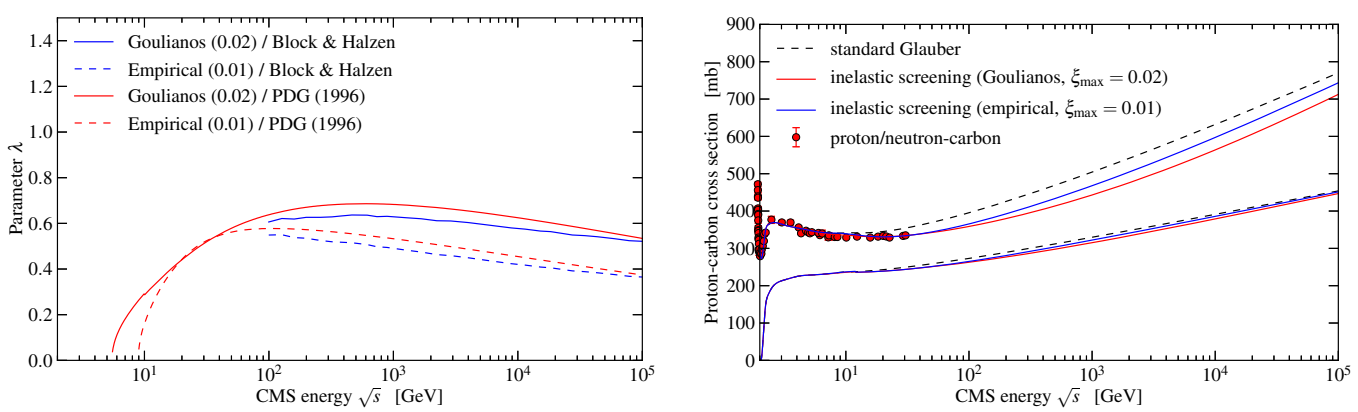

Figure 2. Left panel: energy dependence of the $\lambda$ parameter for different choices of parameterizations and $\xi_{\max }$ (number in brackets). Right panel: comparison of model calculation with proton-nitrogen data ([31] and Refs. therein). The lower set of curves is the production cross-section.
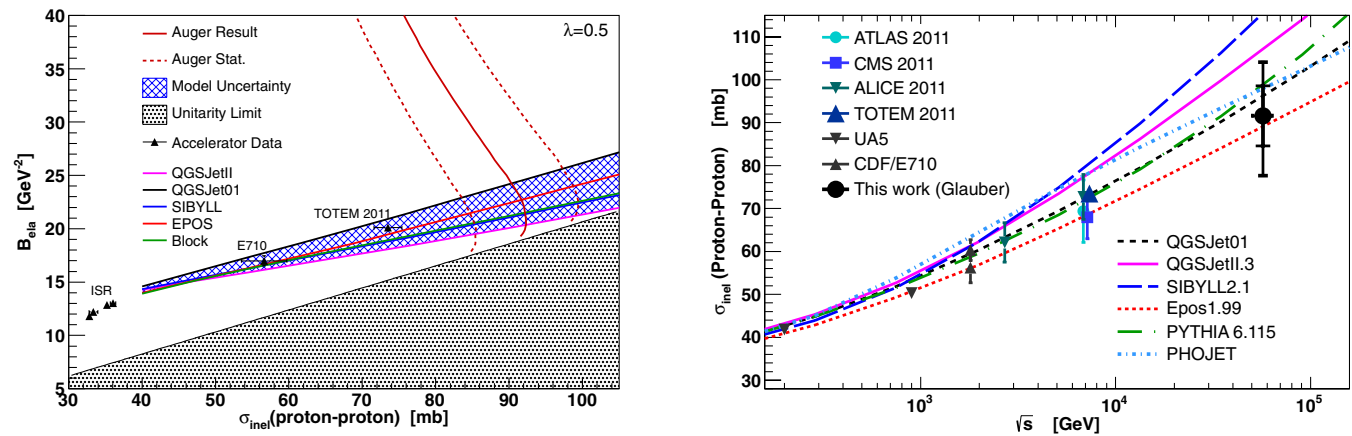

Figure 3. Left panel: correlation of elastic slope parameter, $B_{\mathrm{el}}$, and the inelastic proton-proton cross-section in the Glauber framework. The solid line indicates the parameter combinations yielding the observed proton-air production cross-section, and the dotted lines are the statistical uncertainties. The hatched area corresponds to the predictions by models. Right panel: comparison of derived $\sigma_{p p}^{\text {inel }}$ to model predictions and accelerator data [28]. Here we also show the cross-sections of two typical high-energy models, PYTHIA6 [29] and PHOJET [30]. The inner error bars are statistical, while the outer include systematic uncertainties.

models such as [35] fall within this band. We find that, in the Glauber framework, the inelastic protonproton cross-section is less dependent on model assumptions than the total cross-section. The result for the inelastic and total proton-proton cross-section are

$$
\begin{aligned}
\sigma_{p p}^{\text {inel }} & \left.=\left[92 \pm 7 \text { (stat) }{ }_{-11}^{+9}(\text { sys }) \pm 7 \text { (Glauber) }\right)\right] \mathrm{mb} \quad \text { and } \\
\sigma_{p p}^{\text {tot }} & \left.=\left[133 \pm 13 \text { (stat) }{ }_{-20}^{+17}(\text { sys }) \pm 16 \text { (Glauber }\right)\right] \mathrm{mb} .
\end{aligned}
$$

The systematic uncertainties for the inelastic and total cross-sections include contributions from the elastic slope parameter, from $\lambda$, from the description of the nuclear density profile, and from cross-checking these effects using QGSJetII $[23,36]$. For the inelastic case, these three independent contributions are 1, 3, 5, and $4 \mathrm{mb}$ respectively. For the total cross-section, they are 13, 6, 5, and $4 \mathrm{mb}$. We emphasize that the total theoretical uncertainty of converting the proton-air to a proton-proton crosssection may be larger than estimated here within the Glauber model. There are other extensions of the Glauber model to account for inelastic screening [22, 37] or nucleon-nucleon correlations [38], and alternative approaches that include, for example, parton saturation or other effects $[25,36,39,40]$.

In Fig. 3 (right) we compare our inelastic cross-section result to accelerator data and to the crosssections used in the hadronic interaction models. 


\section{SUMMARY}

We summarized the analysis developed by the Pierre Auger Collaboration to measure cross-sections at $\sqrt{s}=57 \mathrm{TeV}$ center-of-mass energy. The analysis uses high quality hybrid data and measures the fluctuations of proton initiated air-showers in the tail of the $X_{\max }$ distribution at large depths. These fluctuations are related to a value of the proton-air interaction length with the help of simulations. The model-dependence of this conversion is not large, which is also due to the following reasons: Firstly, the fluctuations induced by the primary interaction, and thus of the first interaction point, have the single most important impact on air-shower fluctuations and, secondly, the observable $\Lambda_{\eta}$ is independent of absolute differences between the models and also between the models and data, which can be large.

For comparisons to accelerator data we convert the proton-air cross-section into the inelastic protonproton cross-section. For this purpose we use a Glauber calculation with inelastic screening corrections. The advantage of this calculation is that it has only three relevant free parameters, which are also sufficiently constraint by data to allow a stable extrapolation to cosmic-ray energies. We use the remaining spread in the parameters values of $B_{\mathrm{el}}$ and $\lambda$ as systematic uncertainty of the procedure. It turns out that the inelastic proton-proton cross-section is affected relatively little by systematic uncertainties, while the total proton-proton cross-section is affected much more.

In combination with accelerator data [28] our measurement is compatible with most of the model extrapolations.

\section{References}

[1] The Pierre Auger Collaboaration, Nucl. Instrum. Meth. A 523 (2004) 50

[2] P. Sommers, Astropart. Phys. 3 (1995) 349. B. Dawson et al., Astropart. Phys. 5 (1996) 239

[3] P. Facal for the Pierre Auger Collaboration and D. Garcia-Pinto for the Pierre Auger Collaboration, Proc. 32 ${ }^{\text {nd }}$ ICRC, Bejing, arXiv:1107.4804v1 [astro-ph.HE] (2011)

[4] R. Ellsworth et al., Phys. Rev. D 26 (1982) 336. R. Baltrusaitis et al., Phys. Rev. Lett. 52 (1984) 1380

[5] R. Ulrich, for the Pierre Auger Collaboration, Proc. 32 ${ }^{\text {nd }}$ ICRC, Bejing, arXiv 1107.4804 (2011)

[6] M. Settimo for the Pierre Auger Collaboration, Proc. 32 ${ }^{\text {nd }}$ ICRC, Bejing, arXiv:1107.4804v1 [astro-ph.HE] (2011)

[7] R. Nam et al., Proc. $14^{\text {th }}$ ICRC, Munich 7 (1975) 2258

[8] F. Siohan et al., J. Phys. G 4 (1978) 1169

[9] H. Mielke et al., J. Phys. G 20 (1994) 637

[10] M. Honda et al., Phys. Rev. Lett. 70 (1993) 525

[11] S. Knurenko et al., Proc. 26 ${ }^{\text {th }}$ ICRC, Salt Lake City 1 (1999) 372

[12] K. Belov et al., Nucl. Phys. Proc. Suppl. 151 (2006) 197

[13] M. Aglietta et al., EAS-TOP Collaboration, Phys. Rev. D 79 (2009) 032004

[14] G. Aielli et al., ARGO Collaboration, Phys. Rev. D 80 (2009) 092004

[15] J. Abraham et al., Phys. Rev. Lett. 104 (2010) 091101

[16] R. Ulrich et al., New J. Phys. 11 (2009) 065018

[17] J. Matthews, Astropart. Phys. 22 (2005) 387

[18] R. Ulrich, R. Engel and M. Unger, Phys. Rev. D 83 (2011) 054026

[19] R. Pesce for the Pierre Auger Collaboration, Proc. 32 ${ }^{\text {nd }}$ ICRC, Bejing, arXiv:1107.4809v1 [astroph.HE] (2011)

[20] A. Glushkov et al., Phys. Rev. D 82 (2010) 041101

[21] R. Engel et al., Phys. Rev. D 58 (1998) 014019

[22] N. Kalmykov and S. Ostapchenko, Phys. Atom. Nucl. 56 (1993) 346

[23] S. Ostapchenko, Phys. Rev. D 74 (2006) 014026

[24] E. Ahn et al., Phys. Rev. D 80 (2009) 094003 


\section{UHECR 2012}

[25] K. Werner, Phys. Rev. C 74 (2006) 044902

[26] R. Parsons, C. Bleve, S. Ostapchenko, and J. Knapp, Astropart. Phys. 34 (2011) 832

[27] R. Engel and R. Ulrich, in preparation

[28] ATLAS Collaboration, Nature Commun. 2 (2011) 463. CMS Collaboration, arXiv:1205.3142 [hep-ex] (2012). ALICE Collaboration, presentation at workshop of "Minimum Bias and Underlying Event Working Group”, CERN, 17.6.2011. TOTEM Collaboration, Europhys. Lett. 96 (2011) 21002

[29] G. Schuler, T. Sjostrand, Phys. Rev. D 49 (1994) 2257

[30] R. Engel, Z. Phys. C 66 (1995) 203. R. Engel and J. Ranft, Phys. Rev. D 54 (1996) 4244

[31] SELEX Collaboration, Nucl. Phys. B 579 (2000) 277

[32] R. Glauber, Phys. Rev. 100 (1955) 242. R. Glauber and G. Matthiae, Nucl. Phys. B 21 (1970) 135

[33] M. Good and W. Walker, Phys. Rev. 120 (1960) 1857

[34] K. Goulianos, Phys. Rept. 101 (1983) 169. ALICE Collaboration, arXiv:1202.1727 [hep-ex] (2012)

[35] M. Block, Phys. Rept. 436 (2006) 71

[36] S. Ostapchenko, Phys. Rev. D 81 (2010) 114028

[37] V. Guzey, M. Strikman, Phys. Lett. B 633 (2006) 245

[38] G. Baym, B. Blattel, L.L. Frankfurt, H. Heiselberg and M. Strikman, Phys. Rev. C 52 (1995) 1604

[39] L. Frankfurt, G. Miller, M. Strikman, Phys. Rev. Lett. 71 (1993) 2859

[40] D.R. Harrington, Phys. Rev. C 67 (2003) 064904 